\title{
Surface Acoustic Wave Device Based Wireless Passive Microvalve for Microfluidic Applications
}

\author{
Don W. Dissanayake ${ }^{a}$, Said F. Al-Sarawi ${ }^{a}$ and Derek Abbott ${ }^{a, b}$ \\ ${ }^{a}$ Centre for High Performance Integrated Technologies and Systems (CHiPTec), \\ University of Adelaide, SA 5005, Australia \\ ${ }^{b}$ Centre of Biomedical Engineering (CBME), University of Adelaide, SA 5005, Australia
}

\begin{abstract}
There are vast advantages in the realisation of a SAW device based microvalve in Micro Electro Mechanical Systems (MEMS) and Nano Electro Mechanical Systems (NEMS) such as secure, reliable and low power operation, small size, simplicity in construction and cost effectiveness. In this paper, a Surface Acoustic Wave (SAW) based microvalve that generates micro actuations for microfluidic and similar applications is presented. The microvalve is batteryless and can be actuated wirelessly. The security of the device is enhanced by using a coded SAW correlator that is integrated as part of the microvalve. A theoretical analysis of how the actuation mechanism operates is carried out and simulation results of the new microvalve structure are discussed. The ANSYS simulation tool is used to design and simulate the micro-valve structure. Characteristics of the microvalve actuator in terms of displacement for different operating conditions are also discussed.
\end{abstract}

Keywords: MEMS, Wireless, Passive, Microfluidic, Microvalve, Micropump, SAW, Electrostatic, Actuator, ANSYS

\section{INTRODUCTION}

In modern microfluidic systems, low power operation, small size and secure wireless interrogation are becoming key design issues as these features increase the potential applications of such systems. The nanoliter fluid manipulation is a fundamental function that has a wide range of applications such as drug delivery, fertility control, parallel mixing of photolithographically defined nanoliter volumes, lab-on-a-chip applications, precision manufacturing and miniaturisation of chemical and bio-analysis systems.

One easy and reliable method to incorporate aforementioned features into a microfluidic device is to integrate a SAW device into the microsystem. Based on the published literature, it can be noted that SAW devices are mainly used for wireless communication, sensing and interrogation applications. ${ }^{1-3}$ However SAW devices also being used to develop fluid transfer microsystems such as flexural micropumps and micromachines such as ultrasonic micromotors, micromirrors etc. ${ }^{4,5}$ The fabrication of SAW devices is also becoming easier, especially with microfabrication technologies such as photolithography and X-ray lithography in combination of other well-known processes.

Additionally in MEMS research, SAW device technology has been utilised to design and develop MEMS based microaccelerometers and gyroscopes for military and similar applications. ${ }^{6,7}$ Importantly, it is a well known method to use a sandwich structure of semiconductor on a piezoelectric substrate to form a space-charge coupled SAW devices and SAW convolvers. ${ }^{8}$ Based on the previous research work carried out by the authors, it was proven at the simulation level that a similar approach can be utilised in the design of a SAW based microactuator. ${ }^{9}$ Moreover, the approach used for the actuator design is different to the method used by Varadan et al for their microaccelerometer design. ${ }^{7}$ However the novel aspect of this paper is the demonstration of the possibility of using SAW devices in wireless microactuator applications. In particular, the intention is to highlight the use of SAW devices for microvalve and micropump designs, which can be used in biomedical related microfluidic applications.

Further author information: (Send correspondence to Don W. Dissanayake)

Don W. Dissanayake: E-mail: don.dissanayake@adelaide.edu.au

BioMEMS and Nanotechnology III, edited by Dan V. Nicolau, Derek Abbott, Kourosh Kalantar-Zadeh, Tiziana Di Matteo, Sergey M. Bezrukov, Proc. of SPIE Vol. 6799, 67990H, (2007) · 0277-786X/07/\$18 · doi: 10.1117/12.765011 
In this paper, design and simulation of a SAW device based passive microvalve is discussed. Section 2 provides an important introduction into SAW devices and also shows how the SAW device can be used in a secure manner. In Section 3 a brief description of different actuation mechanisms is provided and the reason to chose SAW device combined with electrostatic actuation is justified. Finite Element Analysis (FEA) simulations carried out in ANSYS tools are depicted in Section 4 and obtained results are discussed in Section 5 which is followed by a conclusion.

\section{SAW DEVICES}

The SAW device comprises a piezoelectric substrate and a set of Inter Digital Transducers (IDT) and/or reflectors as input and output modes to the device ${ }^{1}$ as it is shown in Figure 1. Alternatively a SAW device can be defined as device consisting of thin-film metal structures fabricated on the surface of a piezoelectric substrate. ${ }^{2}$ The purpose of the IDTs on a SAW device is to provide the electromechanical coupling between the electrical signal received (or transmitted) and the mechanical actuation of the piezoelectric substrate material. Generally these IDTs offer a simple and inexpensive means for sensing applications using SAW and integrated micro antennas. The advantages of SAW-IDT design include microfabrication of high quality devices with high accuracy and implementation of differential sensing to compensate for sensor and control unit antenna misalignment. ${ }^{10}$

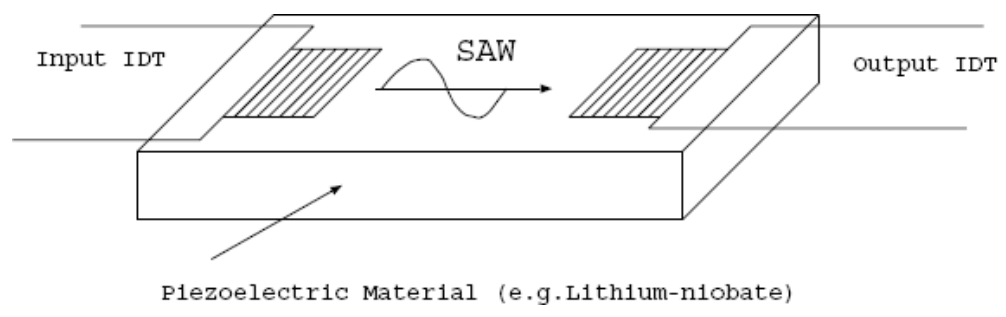

Figure 1. Basic diagram of a SAW device. Consists of piezoelectric substrate, an input IDT and an output IDT. ${ }^{3}$

As it was mentioned above, the key novelty in this proposed microvalve structure is the use of a SAW device as a power transferring mechanism for the microvalve actuation, while operating as a wireless and secure interrogation unit. Hence there is no requirement of a battery and a number of issues related to attaching a battery with an implantable device can be simply ignored.

\subsection{Implementation of SAW Correlator for Secure Actuation}

A coding technique can be used to assimilate the security feature to the SAW device operation. There are different coding sequences such as the Barker code, maximum length code and Golay length code that can be used in SAW devices. These codes are embedded using different finger patterns for input and output IDTs. ${ }^{11}$ The coding of the IDT not only facilitates a secure actuation of the microvalve but also improves the processing gain of the device. The binary weighted Barker sequence is considered for the proposed design as it provides a better actuation of the device due to higher processing gain. ${ }^{11}$ Moreover, a large number of uniquely coded devices can be designed by using combined Barker codes, hence increasing the number of uniquely actuated devices. ${ }^{11,12}$

\section{ELECTROSTATIC ACTUATORS}

\subsection{Different Microactuation Mechanisms}

It is highly important that the microvalve should be extremely reliable and should consist of a better response time to gain a precise control as much as possible especially if the device is aimed at potential biomedical applications. Moreover in an ideal case a higher actuation is highly expected as it increases the flow rate. There are different types of actuation mechanisms that are being used in microfluidic applications at the microscale. The main 
actuation mechanisms are piezoelectric actuation, electrostatic actuation, electromagnetic actuation, pneumatic actuation, Shape Memory Alloy (SMA) actuation, thermo-pneumatic actuation and bimetallic actuation. ${ }^{13}$

Of these mechanisms, piezoelectric actuators are known for low power consumption and high force generation, ${ }^{14}$ though the actuation displacement is comparatively lesser and fabrication requirements are fairly complex. Whereas electrostatic actuators generate a comparatively faster response time and shows a good reliability, even though the small force generated and comparatively smaller stroke are not attractive. ${ }^{15}$ But alternative design measures can be taken to enhance the force and the displacement in electrostatic actuators. Electromagnetic actuators require a large operating voltage to achieve a reasonable displacement. Bimetallic and thermo-pneumatic actuation mechanisms are not considered for this proposed microvalve design as they rely on temperature variations where as the proposed design is based on electric potential variations in the SAW device.

\subsection{MEMS Based Electrostatic Actuation-SAW Device Combined Actuators}

As it was highlighted above, a SAW device is used to incorporate the wireless and batteryless feature to the proposed microvalve structure. As a result a novel and a combined actuation mechanism is needed to allow the linking between the SAW device and the actuator.

Moreover it should be noted that at the output IDT of the SAW device an electric potential is regenerated as a result of the piezoelectric effect in the SAW substrate. By using this feature, a feasible approach is introduced to initiate actuation. In order to generate an electrostatic field, a conductive plate (an equipotential plate) is suspended on top of the output IDT as it can be seen in Figure 2. Due to the electric potential difference between the output IDT and the conductive plate, an electrostatic field is generated and consequently the developed electrostatic force between the conductors causes a deflection in the conductive plate.

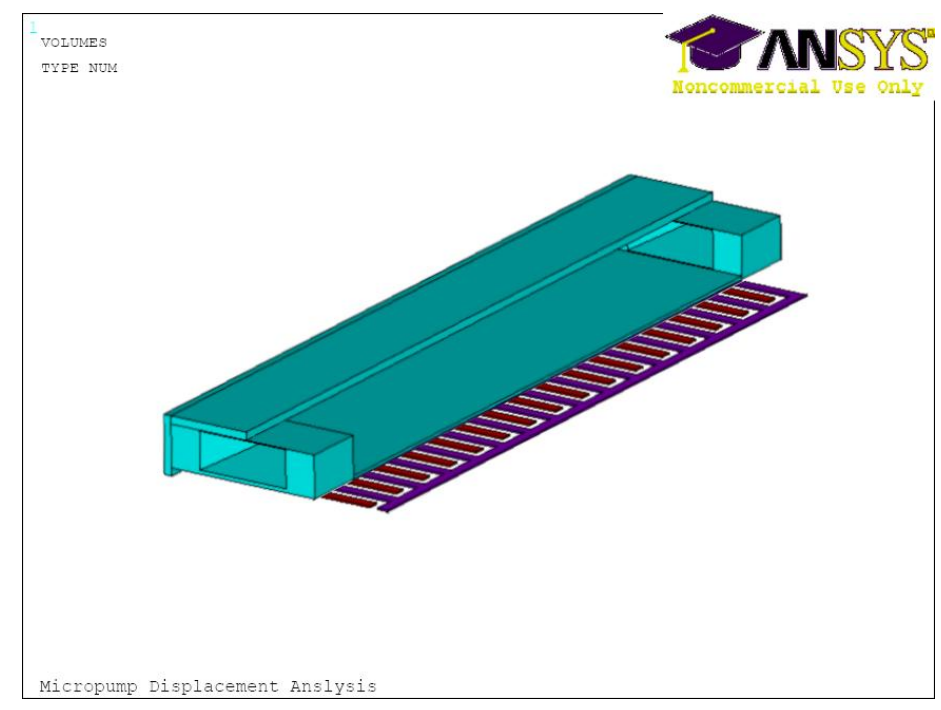

Figure 2. Placement of the pumping chamber on top of the output IDT. The bottom of the pumping chamber is a conductive plate, which is seperated from the output IDT by an air gap.

\subsection{Microvalve and Micropump Structures}

It is beneficial to incorporate the pumping action into the microvalve design as it reduces the dependency of the operation of the device on the surrounding fluid flow as well as causing the fluid to propagate from the inlet to the outlet. A novel micropump structure is designed by integrating the SAW device and the micropump chamber which, causes the microfluidic modulation. As explained in Section 3.2, the pumping chamber is a suspended structure above the output IDT of the SAW device. The chamber consists of two diffusers and a thin plate diaphragm. The diffusers control the inlet and the outlet fluid flow velocities hence introduce the 
flow resistance. When there is a comparatively low pressure inside the chamber, due to diaphragm movement, the fluid is pushed into the chamber through the inlet. The shape of the outlet diffuser is designed to minimise the backward flow from the outlet. Similarly when the pressure inside the chamber is comparatively higher, the fluid gets pushed through the outlet. The inlet diffuser is also designed to minimise the backward flow from the inlet. The diffusers were chosen in this design instead of passive check valves to keep the structure simple and to reduce the fabrication complexity.

\subsection{Proposed microvalve Operation Principle}

A SAW substrate made out of 128-YX-Lithium Niobate and a conductive diaphragm made out of silicon are used to generate an air gap coupled SAW based electrostatic actuator as shown in Figure 3. The device operation is as follows. The input IDT generates Rayleigh waves using inverse piezoelectric effect based on the RF signal that is being fed to the SAW device through a microstrip antenna. The output IDT regenerates the electric signal using the piezoelectric effect of the SAW device. Being an elastic deformation wave on a piezoelectric substrate, the SAW induces charge separation. Thus it carries an electric field with it, which exists both inside and outside the piezoelectric substrate and decays according to Laplace's equation. The conductive plate does not alter the mechanical boundary conditions of the SAW substrate, but causes an equipotential surface and causes the propagating electric potential to be zero at the surface of the conductive plate. The generated electrostatic field between this propagating electric potential wave and the conductive diaphragm on top of the output IDT creates a compulsive and repulsive force between the two. ${ }^{9}$ Since the actuator/diaphragm is a thin flexural plate, it bends as a function of the applied electrostatic field enabling its use as a microactuator in microfluidic applications.

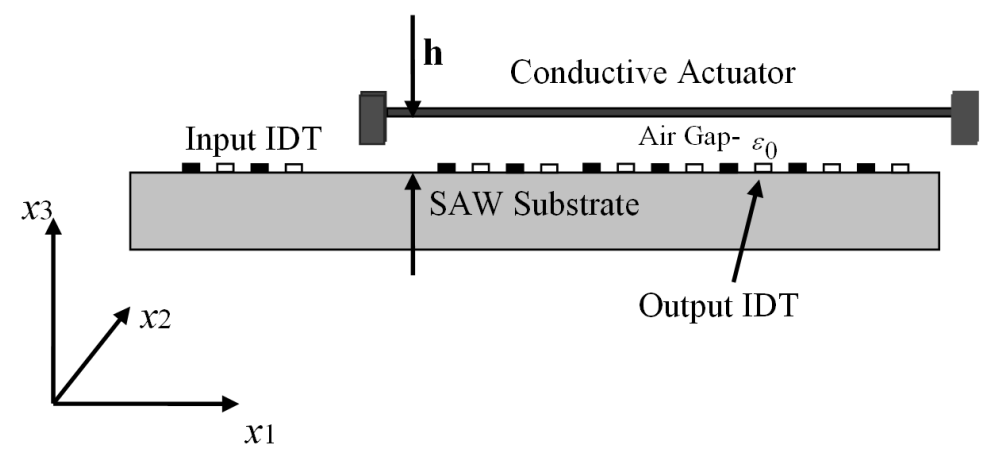

Figure 3. SAW actuator model and boundary conditions. The SAW substrate is the rotated crystal cut to make sure the wave propagation in $x_{1}$ direction and $h$ is the height of the air gap. ${ }^{9}$

\section{FINITE ELEMENT ANALYSIS (FEA)}

In order to analyse the behaviour of the conductive actuator/diaphragm, Finite Element Analysis simulations were carried out using ANSYS simulation tools. ${ }^{16}$ Figure 4 depicts the steps that were followed in the design and modelling of this device. As it can be seen, once the geometry is created, element and material properties are defined for the diaphragm and the air-gap. Next, the created geometry is meshed to a reasonably fine level to accommodate for accurate micro level changes in the structure. Once the geometry is meshed, relevant electric and mechanical boundary conditions are applied by picking either a set of relevant nodes or elements.

After setting the boundary conditions and constrains, a static analysis was carried out mainly to check for the convergence criteria. Once the results are converge to a solution in the static analysis, then a model analysis is carried out to extract the natural frequencies of the conductive actuator. As a result, the operating mode for the actuator can be realised hence the transient analysis is performed for a long enough time period that is dictated by the natural frequency mode of the actuator and the frequency of operation of the SAW device. This 
is an important step in the process as it helps to decide on an optimal end time for transient analysis since the transient simulations generally take a longer time to complete.

\subsection{Design Steps Followed in ANSYS}

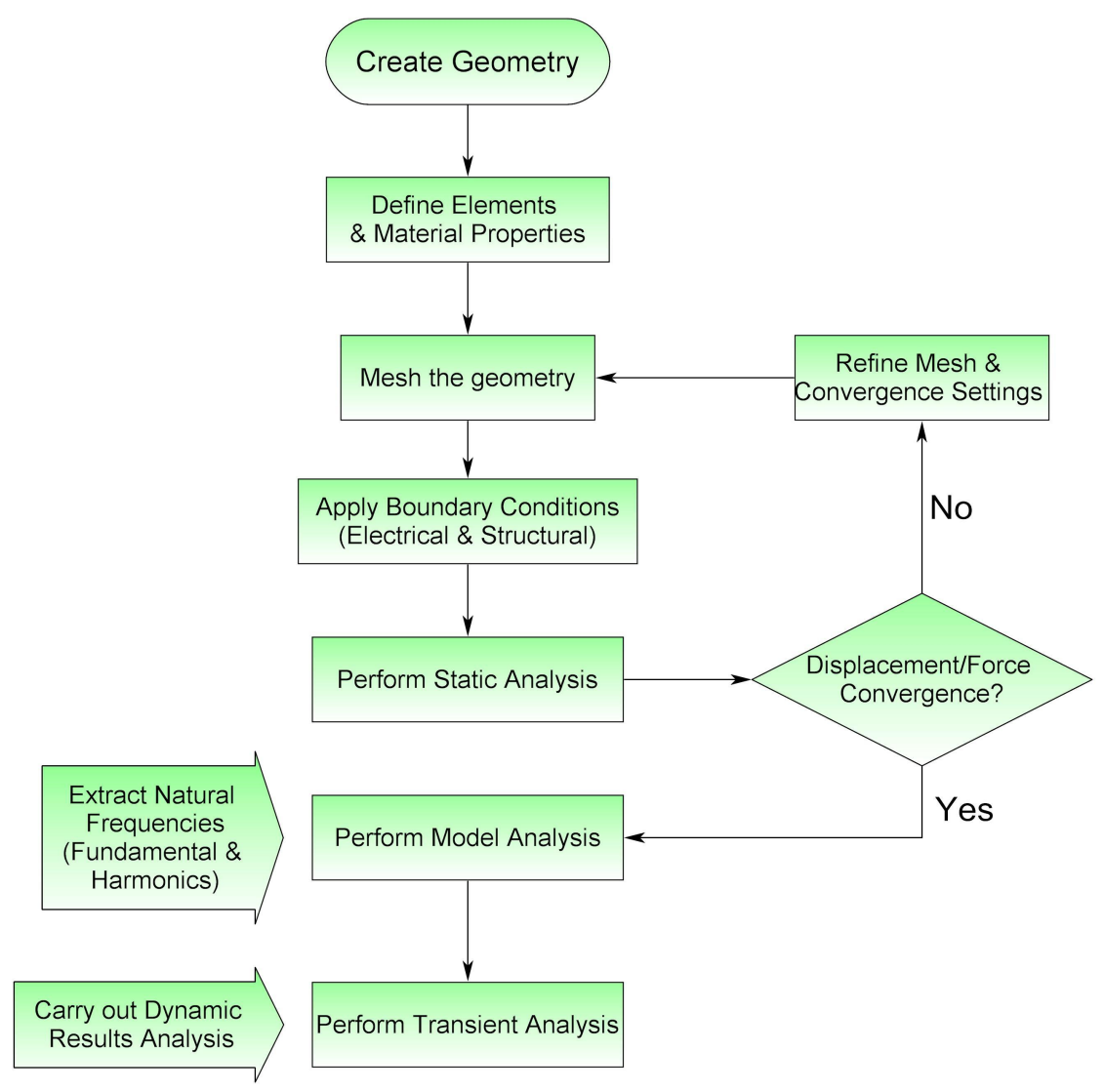

Figure 4. Sequential FEA flow followed in ANSYS.

To simplify the analysis, the performance of the thin conductive plate with a smaller width was initially considered. This is mainly due to the restrictions caused by simulation tool such as node limit and simulation time. As a result only two sides are clamped in the actuator/diaphragm at this stage since it does not provide enough flexibility for a lesser width.

\subsection{Static Analysis}

After necessary boundary conditions and constraints are applied, a static analysis is performed in order to set the convergence settings and develop a basic understanding of the displacement behaviour of the plate as mentioned in the above section. At the preliminary stage, the SAW device and the conductive plate are not analysed as an integrated structure, instead the behaviour of the output IDT is mimicked by coupling a set of nodes that follows the geometry of the output IDT (as shown in Figure 5), then applying a time varying electric potential as given by ${ }^{9}$

$$
\boldsymbol{\Gamma}\left(x_{1}, x_{3}, t\right)=\left\{\begin{aligned}
\Psi, & \text { for } \quad 0 \leq x_{1} \leq \frac{\lambda}{4} \\
\Omega, & \text { for } \quad \frac{\lambda}{4}<x_{1}<\frac{\lambda}{2} \\
-\Psi, & \text { for } \quad \frac{\lambda}{2} \leq x_{1} \leq \frac{3 \lambda}{4} \\
-\Omega, & \text { for } \quad \frac{3 \lambda}{4}<x_{1}<\lambda
\end{aligned}\right.
$$


where $\Psi=\boldsymbol{\Phi}\left(\frac{\lambda}{8}, x_{3}, t\right)$ and $\Omega=\boldsymbol{\Phi}\left(x_{1}, x_{3}, t\right)$ for $\boldsymbol{\Phi}\left(x_{1}, x_{3}, t\right)=\left[\sum_{m} \mathrm{C}_{m} \alpha_{4}^{m} e^{\mathrm{i} k b^{m} x_{3}}\right] . e^{\mathrm{i} k\left(x_{1}-v t\right)}$.

Figure 3 above depicts the reference coordinate system used in these equations. It should be noted that the $\boldsymbol{\Phi}\left(x_{1}, x_{3}, t\right)$ is the electric plane wave component of the SAW. ${ }^{9}$ There $\lambda$ is the wave length of the SAW, $\mathrm{C}_{m}$ are the weighting coefficients, $\alpha_{j}$ values are linear coefficients that depend on the decaying constant $b, k$ is the wave number, $v$ is the SAW velocity in the substrate and $m=1,2,3,4$. The weighting coefficients of these plane waves are chosen to satisfy the mechanical and electrical boundary conditions at the surface of the piezoelectric substrate. ${ }^{9}$

At the simulation level, a 5 Volt sinusoidal electric potential wave with a frequency of $200 \mathrm{MHz}$ was applied to one of the output IDT electrodes and a similar signal with a 180 degree phase difference was applied to the other electrode, since the nature of the Equation 1 is effectively a combination of sinusoidal signals. ${ }^{9}$ The conductive plate was connected to ground so that the plate acts as an equipotential surface.

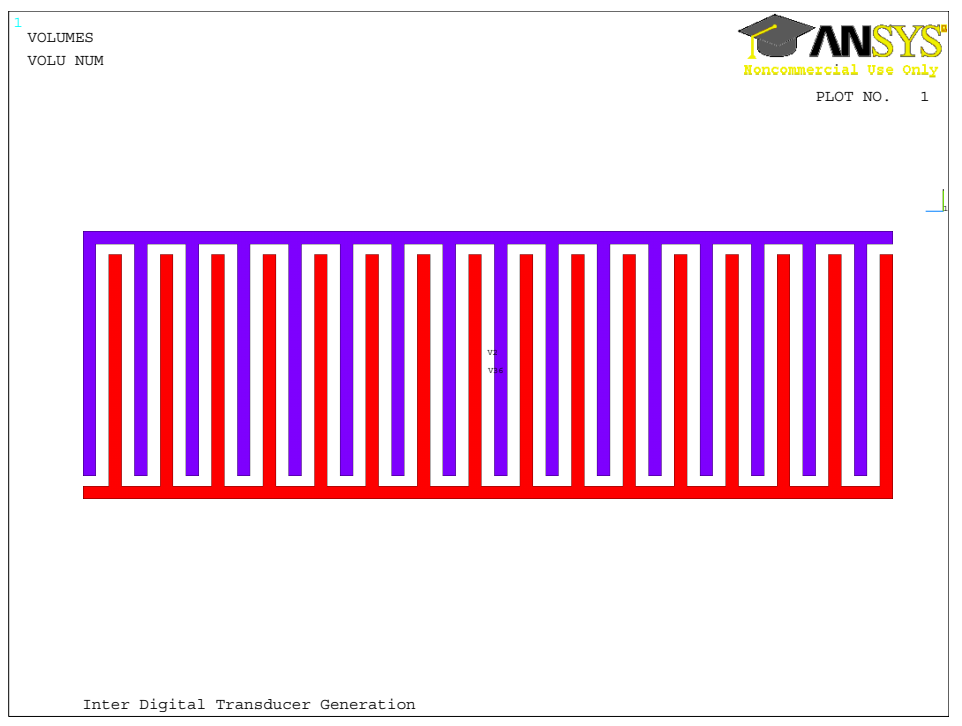

Figure 5. Output IDT electrode pattern to generate the required electrostatic field.

\subsection{Transient Analysis}

Once the static analysis is completed, following a model analysis, more importantly a transient analysis is carried out in order to study the dynamic behaviour of the SAW device based electrostatic actuator. This is more substantial in investigating the operating frequency of the conductive plate. Since the SAW frequency is in the range between $100 \mathrm{MHz}-1 \mathrm{GHz}$ it is crucial to verify the effective operating frequency of the conductive plate. Because of the time varying electrostatic field, the operating frequency of the actuator is much less than that of the SAW frequency. A higher vibration frequency could cause a structural instability and could also affect the lifetime of the microactuator which leading to reduction of the device reliability. Moreover it should be noted that when a conductive beam is subject to a dynamically changing electrostatic field, the displacement behaviour needs to be calculated analytically using an iterative process. At the initial stage, ANSYS based FEA method greatly helps to analyse such a dynamic behaviour as the ANSYS solvers are equipped with analytical algorithms. 


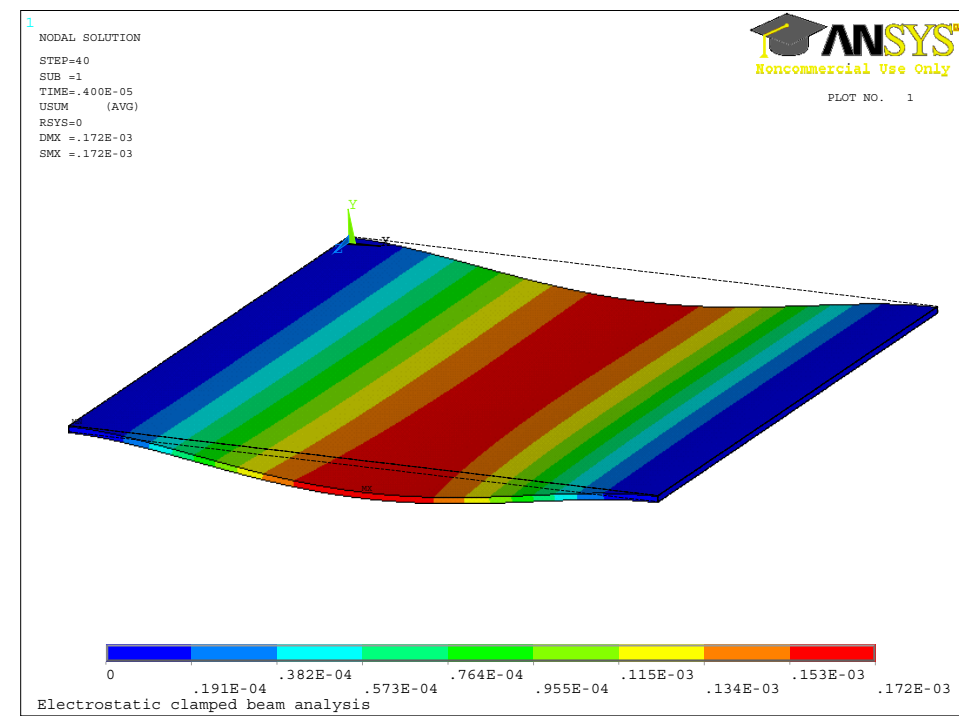

Figure 6. A contour plot of the displaced diaphragm. Only two sides are clamped at this stage as the diaphragm width is less than the actual dimension.

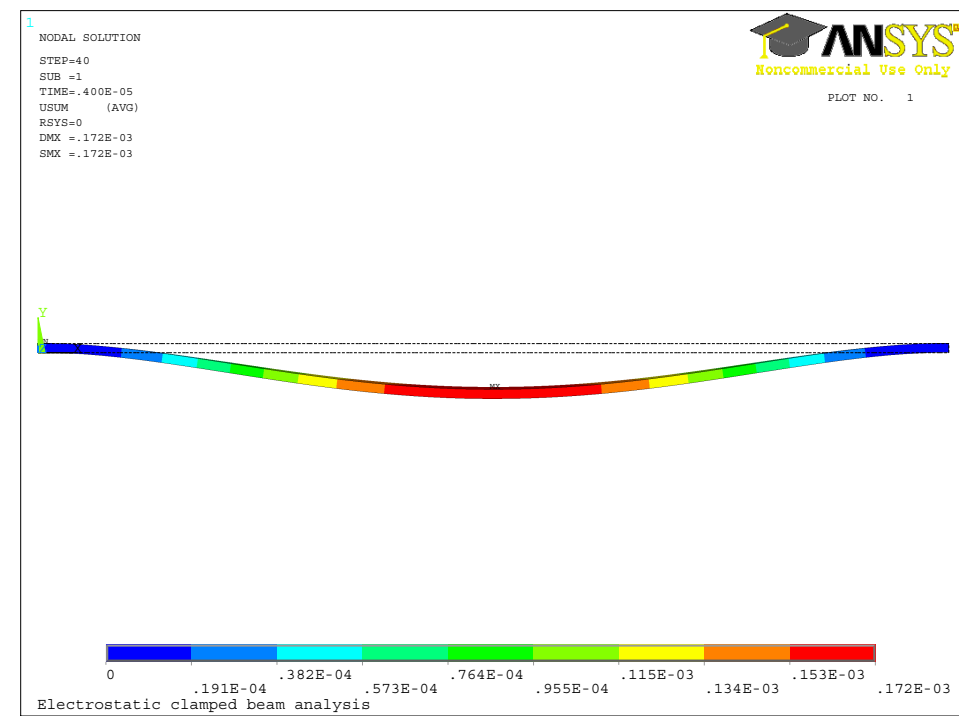

Figure 7. A side view of the displaced diaphragm. Thinner diaphragm is analysed to achieve higher displacements.

\section{SIMULATION RESULTS ANALYSIS}

A conductive plate with different lengths $(400 \mu \mathrm{m}-2000 \mu \mathrm{m})$ is analysed at simulation level. This section depicts the transient simulation results carried out for a conductive plate with dimensions $1000 \mu \mathrm{m} \times 2 \mu \mathrm{m} \times 10 \mu \mathrm{m}(\mathrm{L}$ $\times \mathrm{H} \times \mathrm{W}$ ). Moreover the input electrical signal is similar to the one that was used for the static analysis. Figure 6 depicts a contour plot of the displaced diaphragm and Figure 7 shows a side view of the displaced diaphragm. As can be seen from these simulations, the micro displacements are successfully obtained using this method. Figure 8 shows the mid-beam and the quarter-beam displacement variation over the time (250-time-periods). 
Based on authors' previous work, it was shown that beam displacements up to $2 \mu \mathrm{m}$ can be achieved using SAW device based actuation. ${ }^{9}$ After carrying out a few different transient analysis simulations with different end times and comparing the obtained transient displacements with the static displacements achieved, it is proven even after 250-time-periods still the dynamic displacement is not showing any periodic nature but in the process of gaining more displacement. Based on the results obtained so far, it is evident that the actual vibration frequency of the conductive plate is a very much scaled down version of the SAW operating frequency. Interestingly such actuator behaviour will boost the controllability of the micropump with increased reliability. Figure 9 depicts a vector plot of the displaced conductive actuator. Because of the time varying nature of the electrostatic field that is generated by the output IDTs, the actuator consists of flexural displacement component as it can be seen from the vector plot.

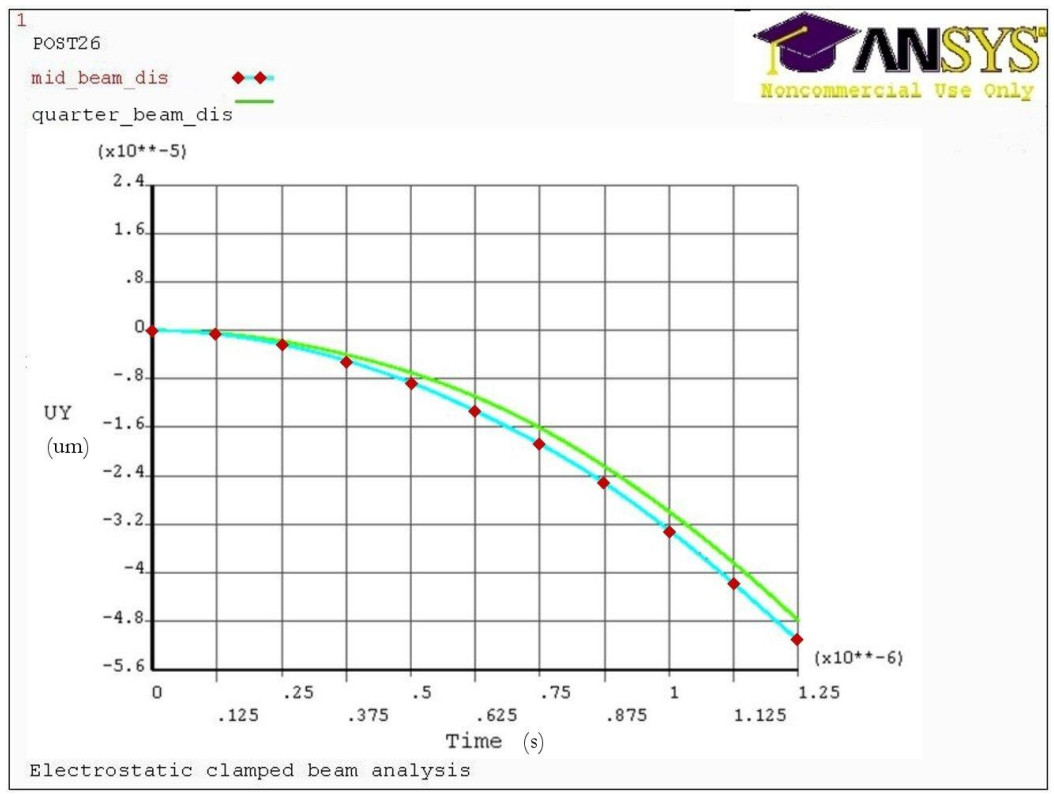

Figure 8. Displacement vs Time plot of the mid-beam of the conductive plate. Analysis carried out for $250 \times T$, where $T$ is the time period of the SAW signal.

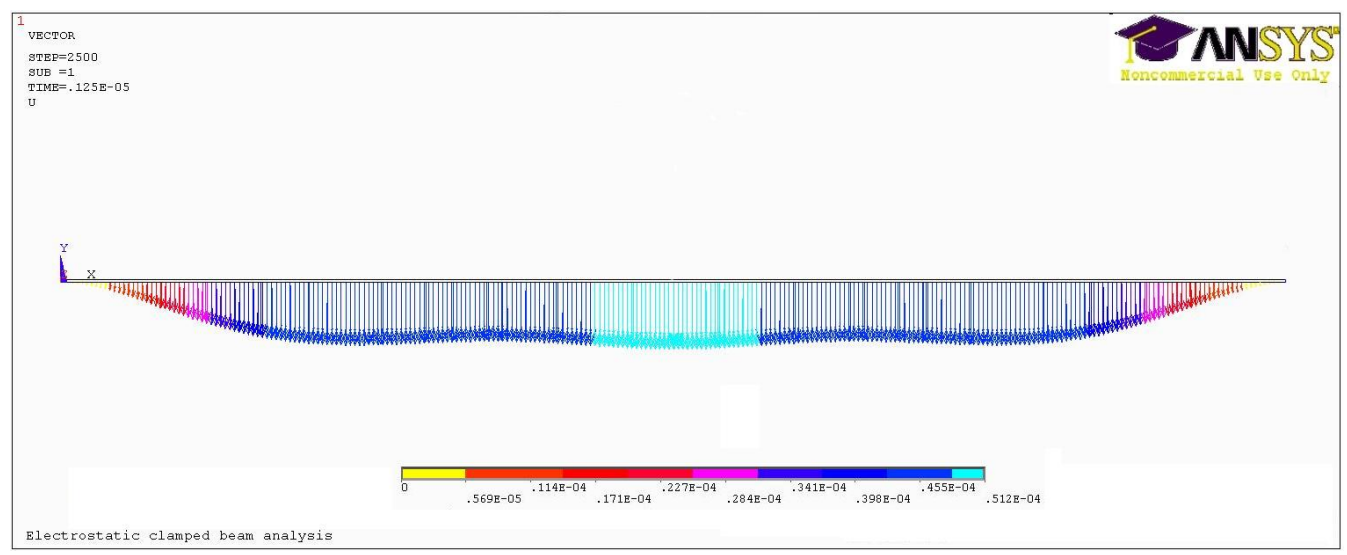

Figure 9. A Vector plot of the displaced conductive plate obtained after the transient analysis. Vector shows nodal displacements of the diaphragm. 


\section{CONCLUSION}

A novel method to develop a wireless and a battery-less passive microvalve is introduced in this paper. The importance of a microfluidic device with such features for biomedical applications is highlighted and a FEA analysis carried out using ANSYS tools is also presented. Especially, the FEA simulations are carried out according to a structured method in order to optimise the simulation time while achieving better quality results. Static analysis results showed that the conductive plate is capable of achieving micro-displacements using the SAW device combined electrostatic actuation mechanism. A significant down conversion of the vibration frequency of the diaphragm is clearly observed from the transient analysis results, even though the results are not sufficient to determine the vibration frequency.

As a result of this research work, some important future research is identified in order to improve the design and to provide a complete design for the microvalve. Design of the diaphragm to be more flexible by carefully choosing materials that have best suited mechanical properties for higher flexibility, while maintaining the structural stability, is one important step in this process. As a part of this, investigation of polymer based materials is an important milestone as much research takes place for a variety of conductive polymer materials. The possibility is high for such materials to be readily available for low cost in the future. From the structural point of view, designing a corrugated diaphragm will reduce the stiffness and hence will provide more flexibility for micro displacements. After the structural design is completed, then microfluidic analysis needs to be carried out in order to analyse the microvalve operation while different fluids are present and to counter act on the effects

such as back-pressure and fluid leakages. Once the microvalve device is tested at simulation level, a prototype is expected to be fabricated in order to demonstrate concept of operation in a physical environment.

\section{ACKNOWLEDGMENTS}

The authors would like to thank the Australian Research Council (ARC) and the School of Electrical and Electronic Engineering (University of Adelaide, Australia) for providing the necessary funding, facilities and the support to carry out this research.

\section{REFERENCES}

1. C. K. Campbell, Surface Acoustic Wave Devices for Mobile and Wireless Communications, San Diago, Califonia: Academic Press, 1998.

2. J. W. Gardner, V. K. Varadan and O. O. Awadelkarim, Microsensors, MEMS, and Smart Devices, Tsinghua University Press, Beijing, 2001.

3. I. Pitz, L. T. Hall, H. J. Hansen, V. K. Varadan, C. D. Bertram, S. Maddocks, S. Enderling, D. Saint, S. F. Al-Sarawi and D. Abbott, "Trade-offs for Wireless Transcutaneous RF Communication in Biotelemetric Applications," Proc. of SPIE-Biomedical Applications of Micro- and Nanoengineering 4937, pp. 307-318, November 2002.

4. A. Wixforth, "Acoustically driven planar microfluidics," Superlattices and Microstructures 6, pp. 389-396, 2003.

5. C. J. Strobl, Z. V. Guttenberg and A. Wixforth, "A Nano- and pico-dispensing of fluids on planar substrates using SAW," IEEE Transactions on Ultrasonics, Ferroelectrics, and Frequency Control 51(11), pp. 14321436, 2004.

6. V. K. Varadan and V. V. Varadan, "Microsensors, micromechanical systems (MEMS), and electronics for smart structures and systems," Smart Materials and Structures 9, pp. 953-972, 2000.

7. H. Subramanian, V. K. Varadan, V. V. Varadan and M. J. Vellekoopz, "Design and fabrication of wireless remotely readable MEMS based microaccelerometers," Smart Materials and Structures 6(6), pp. 730-738, 1997.

8. L. B. Milstein and P. Das, "Surface Acoustic Wave Devices," IEEE Communications Magazine 17, pp. 2533, 1979.

9. D. W. Dissanayake, S. Al-Sarawi and D. Abbott, "Surface Acoustic Wave Device Based Electrostatic Actuator for Microfluidic Applications," 2nd International Conference on Sensing Technology, pp. 381-386, November 2007. 
10. A. S. Sezen, S. Sivaramakrishnan, S. Hur, R. Rajamani, W. Robbins and B. J. Nelson, "Passive Wireless MEMS Microphones for Biomedical Applications," Journal of Biomedical Engineering 127, pp. 1030-1034, 2005.

11. A. C. Tikka, S. Al-Sarawi, D. Abbott, M. S. K. Wong and J. D. Schutz, "Improving the Security and Actuation of Wireless Controlled Microvalve," Proc. of SPIE-Smart Structures, Devices, and Systems III 6414, January 2007.

12. A. C. Tikka, S. Al-Sarawi and D. Abbott, "SAW parameter extraction using Finite Element Analysis," 2nd International Conference on Sensing Technology, pp. 393-398, November 2007.

13. D. W. Dissanayake, A. C. Tikka, S. Al-Sarawi and D. Abbott, "Radio frequency controlled microvalve for biomedical applications," Proc. of SPIE-Smart Materials IV 6413, 2007.

14. R. Duggirala, S. Son and A. Lal, "A Pyroelectric-Piezoelectric Valve for Integrated Microfluidics," Proc. of IEEE International Solid-State Sensors and Actuators 2, pp. 1554-1557.

15. N. T. Nguyen, X. Huang and T. K. Chuan, "MEMS-Micropumps: A Review," Transactions of the ASME Journal of Fluids Engineering 124, pp. 384-392, June 2002.

16. ANSYS Incorporation, "Home page." visited on 21/11/2007. 